analysis suggested the inconsistency is not due to any single recommendation source. These findings held whether considering all recommendation sources or only CPGs. One recommendation source reported patient or public involvement. Six included very general information about how to include patients in individual decision-making, and three provided direct-to-patient guidance. Two made tools available to help patients participate in individual decision-making, one suggesting an existing tool, and the other integrating the tool within the POC recommendation.

Conclusions Hypertension is a common chronic condition with widespread expectations surrounding guideline-based care, but CPGs have high degrees of inconsistency. Further investigation should determine the reasons for inconsistency, the implications for recommendation development, and the role of synthesis across recommendations for optimal guidance of clinical care.

Consideration of a patient's values and preferences is a fundamental part of practicing evidence-based medicine. Therefore, public and patient involvement is encouraged in CPG development just as shared decision-making is encouraged in clinical practice. With a substantial proportion of hypertension management guidance being weak or inconsistent, shared decision-making could replace algorithmic instructions as a primary framework for an approach to healthcare, but this will require development of patient decision aids and workflow support tools to make it practical.

\section{OPEN ACCESS BUDGET TOOLS FOR THE PLANNING OF RANDOMISED CONTROLLED TRIALS: A SCOPING REVIEW}

\begin{abstract}
1,2Benjamin Speich, ${ }^{2}$ Viktoria Gloy, ${ }^{3}$ Nadine Schur, ${ }^{2}$ Lars G Hemkens, ${ }^{3}$ Matthias Schwenkglenks, ${ }^{2}$ Matthias Briel. ${ }^{1}$ Centre for Statistics in Medicine, Nuffield Department of Orthopaedics, Rheumatology and Musculoskeletal Sciences, University of Oxford, UK, Oxford, UK; ${ }^{2}$ Basel Institute for Clinical Epidemiology and Biostatistics, Department of Clinical Research, University of Basel and University Hospital Basel, Switzerland, Basel, Switzerland; 'Institute of Pharmaceutical Medicine, University of Basel, Basel, Switzerland, Basel, Switzerland
\end{abstract}

10.1136/bmjebm-2019-EBMLive.40

Objectives Well conducted randomised controlled trials (RCTs) generate the most trustworthy evidence when newly developed or already existing clinical interventions are evaluated. However, RCTs require substantial resources and the costs seem to be increasing over time. As a result, the number of independent investigator-initiated RCTs decreased and a considerable proportion of RCTs are prematurely discontinued due to recruitment delays. Especially in academic research, it is common that clinical studies are underfinanced or that the compensations paid by funders or industries do not cover the actual costs. Therefore, tools for investigators to plan their budgets accurately are essential for the successful conduct of an RCT. In this scoping review we aimed to give an overview of the publicly available budget planning tools.

Method We systematically searched Medline, EMBASE (both via Ovid) and EconLit from inception until May 2018. Additionally, two reviewers conducted an internet search between June and October 2018. We included any tools or cost templates and categorised them if they were primarily intended to (i) plan a budget for an entire RCT, (ii) plan a budget for a separate centre participating in a RCT, or (iii) monitor costs during the conduct of an RCT. From all tools we assessed if they considered direct costs (fixed costs and variable costs) as well as indirect costs, and if they were user tested or validated in any form.

Results We identified 25 tools which were included (i.e. two from the literature search and 23 from the internet search). Of those, 22 tools consisted of programmed Microsoft excel sheets. Seven tools were developed to plan the budget for an entire RCT, 17 tools helped to calculate budgets for a separate study centre participating in an RCT, and the purpose of one tool was to monitor ongoing costs of RCTs. Direct costs, consisting of fixed costs and variable costs were considered by 25 and 21 tools, respectively. Indirect costs were considered by 19 tools. Overall, 18 tools considered all three of these cost aspects. Of the seven tools which can be used to plan costs of an entire RCT, only two included all three relevant cost aspects. Overall, we identified a description of user testing or validation for two tools only.

Conclusions A variety of freely accessible budget planning tools for RCTs exist. Most of the tools were developed internally by different institutions and were not published in a scientific journal. Often it remained unclear if they underwent any form of validation or if they can also be applied in a useful way by researchers who are not part of the institution which developed the tool. We did not find any evidence during this project that a single tool was broadly applied for planning RCT budgets. The fact that many different organisations put effort in developing a budgeting tool shows that the need for accurate planning of RCT budgets was recognised at many levels. Identifying or creating a user-friendly tool which can be used flexibly for different RCTs in different settings should therefore be a research priority.

\section{LONGITUDINAL CURRICULUM FOR CERTIFIED TRAINING IN EVIDENCE-BASED MEDICINE: CERTIFIED EVIDENCE- BASED MEDICINE PRACTITIONER. (CEBMP)}

${ }^{1}$ Izhar Hasan, ${ }^{2}$ Uzair Hasan, ${ }^{3}$ Salman Habib Abbasi, ${ }^{4}$ Shankar Srinivasan, ${ }^{5}$ Babar Rao. ${ }^{1}$ MDACCESS, Princeton, USA; ${ }^{2}$ MDACCESS, Princeton, NJ, USA; ${ }^{3} H i$ Tech Medical college, Taxilla, Pakistan; ${ }^{4}$ Department of Health informatics, Rutgers university, Newark, NJ, USA; ${ }^{5}$ Robert wood Jhonson Medical school, Somerset, New Jersey, USA

\subsection{6/bmjebm-2019-EBMLive.41}

Objectives Training in evidence based medicine practice is a mandatory core competency of practice based learning and improvement (PBLI) of ACGME standards.Reflective medical education is a triggering point for self-directed learning at the point of care in wards, clinics and in operating rooms to capture patient specific clinical queries. Both these skills can be taught through a longitudinal curriculum which emphasizes on patient and learner-centered education. However, there is no formal longitudinal curriculum to teach reflective learning and evidence-based medicine to medical students and trainees in a clinically integrated learning environment during multiyear training. Web based certification training is a growing trend to demonstrate commitment to professionalism, and offers a formal training to meet the standards for self-directed learning for professional development

Method Our objective is to implement a web based longitudinal curriculum for certified training in evidence-based medicine. We plan to customized specialty based longitudinal training in evidence based medicine practicing skills. Furthermore, our goal is to identify the areas of insufficient or poor evidence in each specialty to emphasize the training in skills 